\title{
Effects of self- and cross-phase modulation on the spontaneous symmetry breaking of light in ring resonators
}

\author{
Lewis Hill ${ }^{1,2},{ }^{*}$ Gian-Luca Oppo ${ }^{1}$, Michael T. M. Woodley ${ }^{2,3}$, and Pascal Del'Haye ${ }^{2}$ \\ ${ }^{1}$ Department of Physics, University of Strathclyde, Glasgow G4 ONG, United Kingdom \\ ${ }^{2}$ National Physical Laboratory, Hampton Road, Teddington, TW11 OLW, United Kingdom \\ ${ }^{3}$ School of Physics, Heriot-Watt University, Edinburgh, EH14 4AS, United Kingdom
}

\begin{abstract}
Spontaneous symmetry breaking can occur in the powers of two optical modes coupled into a ring resonator, described by a pair of coupled Lorentzian equations, and featuring tunable self- and cross-phase modulation terms. Investigated is a wide variety of nonlinear materials by changing the ratio of the self- and cross-phase interaction coefficients. Static and dynamic effects range from the number and stability of stationary states to the onset and nature of oscillations. Minimal conditions to observe symmetry breaking are provided in terms of the ratio of the self-/cross-phase coefficients, detuning, and input power. Different ratios of the nonlinear coefficients also influence the dynamical regime, where they can induce or suppress bifurcations and oscillations. A generalised description on this kind is useful for the development of all-optical components, such as isolators and oscillators, constructed from a wide variety of optical media in ring resonators.
\end{abstract}

PACS numbers:

\section{INTRODUCTION}

Originally proposed in 1987, the Lugiato-Lefever equation (LLE) [1] has been used to model a variety of nonlinear optical systems [2]. One of the equation's major successes has been in describing light propagating in fibre loops and microring resonators featuring Kerr media - materials in which the refractive index is modified by the intensity of the propagating light. While the original model described spatio-temporal dynamics in the plane transverse to the direction of propagation, a later model for purely temporal effects [3] has been demonstrated to be mathematically equivalent $[4,5]$.

Coupled LLEs have been used to describe normalised leftand right-circularly-polarised field envelopes, $E_{ \pm}$, in FabryPerot or ring cavities [6]. This system of two coupled LLEs is given by

$$
\begin{aligned}
\frac{\partial E_{ \pm}}{\partial t}=E_{I n}-E_{ \pm}-i \eta \theta E_{ \pm} & +i a \nabla^{2} E_{ \pm} \\
& +i \eta\left(A\left|E_{ \pm}\right|^{2}+B\left|E_{\mp}\right|^{2}\right) E_{ \pm},
\end{aligned}
$$

where $\theta$ denotes the cavity detuning - the difference between the input field's frequency and the closest cavity resonance frequency, $a$ describes the transverse diffraction strength, $E_{\mathrm{In}}$ is the input pump envelope, $\eta= \pm 1$ indicates either a selffocusing, +1 , or self-defocusing medium, -1 , respectively, and $\nabla^{2}$ is the transverse Laplacian. The one-dimensional case of Eq. (1), with diffraction replaced by dispersion, describes the propagation of two optical field components in ring resonators. In the case of continuous wave (CW) inputs and negligible dispersion, Eq. (1) describe either two co-propagating fields with left and right circular polarizations (see Fig. 1(a)) or two counter-propagating fields with equal linear polarization (see Fig. 1(b)). The coupling constants $A$ and $B$ are related to the third-order nonlinear susceptibility tensor, $\chi^{(3)}$,

\footnotetext{
*Electronic address: lewis.hill@strath.ac.uk
}
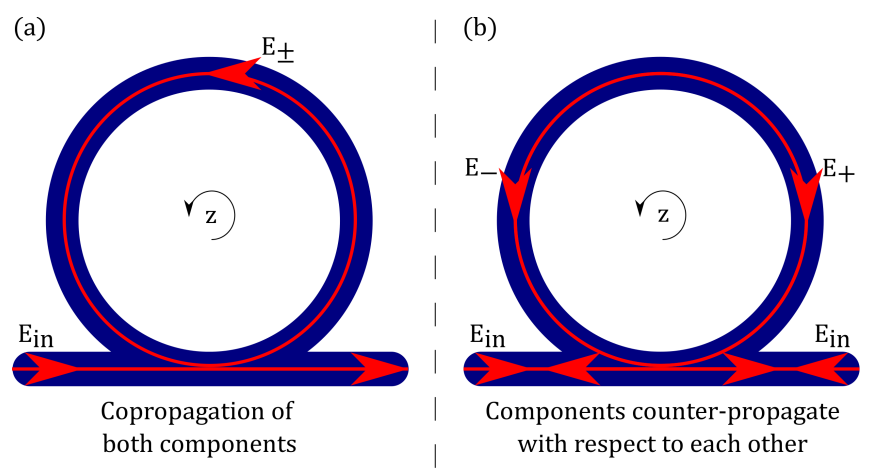

FIG. 1: Ring resonator setups, showing: (a) copropagation of two light components with left and right circular polarisations and (b) the counter-propagation of two light beams, linearly polarised along the same axis.

and describe the strengths of self- and cross-phase modulation, respectively - the former is the change in refractive index induced by one optical mode on itself, and the latter is the change induced by the other mode. The values of these two coefficients are of great interest because their relative magnitudes and signs vary across a large number of different experimental configurations. These situations include light propagating through dielectrics, optical fibres, Kerr liquids (such as carbon disulphide, benzene, toluene, and certain liquid crystals), engineered structures such as periodically-poled lithium niobate, as well as experiments featuring atomic vapours. We provide a comprehensive theory of spontaneous symmetry breaking in the intensity of two distinct modes, across a variety of different experimental contexts, by considering variations of the ratio $B / A$ - the central parameter of the investigations.

By restricting the solution set of $E_{ \pm}$in Eq. (1) to being both stationary and homogeneous, and then multiplying each ele- 
ment by its complex conjugate, one obtains

$$
P_{1,2}=\frac{I}{1+\left(\theta-A P_{1,2}-B P_{2,1}\right)^{2}},
$$

where we have set $\left|E_{+}\right|^{2}=P_{1},\left|E_{-}\right|^{2}=P_{2}$ and $E_{\text {In }}^{2}=I$ for the ease of notation. This particular solution corresponds to two coupled Lorentzian equations - mathematically identical to those that describe two normalised, linearly polarised, counter-propagating stationary fields in ring resonators [712], Fig. 1(b). Of course, mathematical equivalence does not necessarily imply physical equivalence. In the counterpropagating case, $E_{ \pm}$are the two counter-propagating field envelopes and the coupling constants $A$ and $B$ now depend on the formation of an index grating generated by the two fields, rather than on $\chi^{(3)}$ as with the polarisation equations [13-16].

Equation (2) can be understood as the homogeneous stationary solution set of any system described by two coupled LLEs, such that many of the subsequent results of this paper can be applied not only to both the counter-propagating and polarisation cases, but to other physical systems, too.

One fascinating phenomenon that arises from a system of two coupled Lorentzian equations, such as Eq. (2), is spontaneous symmetry breaking $[8,11]$. We first extend the investigation of the onset of symmetry breaking in ring resonators to a variable ratio of the self- and cross-phase modulation terms, $B / A$, in Section II. We then identify the steady-state characteristics of the symmetry breaking for variable $B / A$ in Section III. Sections IV and V are devoted to an analytical stability analysis and dynamical behaviour via numerical integration, respectively. In the latter case, we ascertain how varying the cross-coupling strength between the two fields alters the temporal instability of the system, thereby encouraging or suppressing deterministic chaos. Our conclusions are summarised in Section VI.

Symmetry breaking phenomena have a wide range of applications in nonlinear optics: to enhance the Sagnac effect $[7,11]$; to realise optical isolators, circulators [10], and oscillators [12]; and to enhance near-field detectors [17]. A possible area of further application is the generation of temporal cavity solitons (TCS) for data storage and in the generation of frequency combs. There is enormous interest in extending the range and realisation of TCS due to their diverse utility in fields such as precision metrology, gas sensing, arbitrary optical waveform generation, and telecommunications [18].

\section{SPONTANEOUS SYMMETRY BREAKING}

Spontaneous symmetry breaking of two modes in an optical ring resonator manifests itself as unequal coupling of the two input powers into the resonator. Consequently, we will refer to spontaneous symmetry breaking of the 'coupled powers'. This was first predicted theoretically in Ref. [8], and has since been experimentally observed in Ref. [9, 10] for counter-propagating fields, whilst the polarisation case is discussed in Ref. [6, 19, 20], and experimentally in Ref. [21, 22].

Spontaneous symmetry breaking in the coupled Lorentzian system can be visualised in a number of ways. One way is
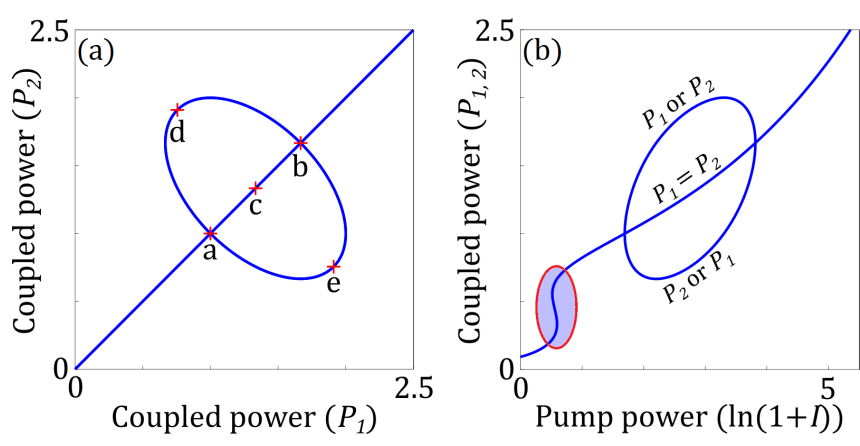

FIG. 2: Different graphical representations of spontaneous symmetry breaking when scanning pump power, $I$, shown here for $A=1, B=$ 2 and $\theta=2$. (a) Coupled powers are plotted against each other, Eq. (3). The points $a$ and $b$ indicate the opening and closing of the symmetry-broken bubble given by Eq. (12). Point $\mathrm{c}$ is the point at which symmetry breaking occurs at the detuning limit, see Eq. (13). The maximum coupled power difference can be found at points $d$ and e, see Eq. (15). (b) The coupled powers are plotted against input power. Note the visible presence of the 'symmetric bistability' (the highlighted S-shaped curve), but also the loss of some symmetries seen in (a).

to eliminate the explicit dependence on the pump power, $I$, by rearranging Eq. (2) such that the two expressions are each made equal to $I$. They may then be solved simultaneously as

$$
P_{1}\left[1+\left(\theta-A P_{1}-B P_{2}\right)^{2}\right]=P_{2}\left[1+\left(\theta-A P_{2}-B P_{1}\right)^{2}\right]
$$

This solution is plotted in Fig. 2(a), and corresponds to a 'scan' with respect to the pump power, $I$, shown in Fig. 2(b). The 'symmetric' solution line features as a simple $P_{1}=P_{2}$ relationship, and the spontaneous emergence of the symmetrybroken solution line is characterised by an ellipse. The point at which symmetry-broken solutions arise is known as the "symmetry breaking bifurcation point', whereas the point at which they disappear is the 'inverse bifurcation point'.

It has been shown that, in the case of $A=1, B=2$, the symmetric solution line between the bifurcation points is unstable, and so, if the system is subject to a perturbation, such as noise, it will evolve towards the stable symmetry-broken solution line [8]. This is an extremely useful result, since it means that the two observed field envelopes will no longer circulate with equal intensity - one field envelope will become dominant, whilst the other is quenched. This behaviour is central to the applications mentioned previously.

Figure 2(a) is the counterpart of Fig. 2(b), originally reported in Ref. [8]. In different ways, they both illustrate the symmetry breaking by scanning the input power. An informative advantage of Fig. 1(b) comes from its ability to show the 'symmetric bistability' - highlighted by a red ring. This region is present in Fig. 2(a), but is hidden within the symmetric solution line. The advantage of Fig. 2(a), however, comes from its additional symmetry, which can allow for mathematical simplifications in the derivations of later results.

It is also possible to observe symmetry breaking when scanning the cavity detuning rather than the pump power. This can be done by employing a similar method to above - by rear- 

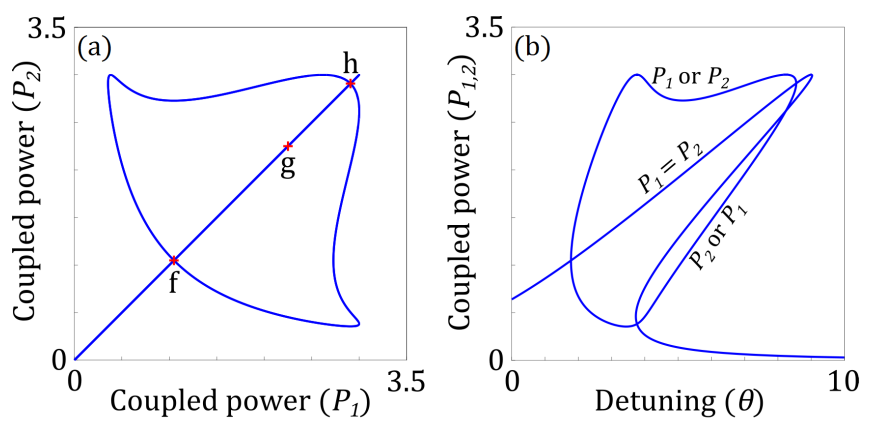

FIG. 3: Different graphical representations of spontaneous symmetry breaking when scanning detuning, $\theta$, shown here for $A=1, B=2$ and $I=3$. (a) Coupled powers are plotted against each other, Eq. (4). The points $f$ and $h$ indicate the symmetry breaking bifurcation pair for a detuning scan - see Eq. (17). At point g, the bubble emerges at the intensity limit - see Eq. (16). (b) Coupled envelope powers are plotted against detuning, $\theta$. Note again the loss of symmetry between (a) and (b).

ranging Eq. (2) such that the two expressions are in terms of $\theta$; they can again be solved simultaneously, eliminating $\theta$,

$$
A P_{1}+B P_{2} \pm \sqrt{\frac{I}{P_{1}}-1}=A P_{2}+B P_{1} \pm \sqrt{\frac{I}{P_{2}}-1},
$$

where each \pm is independent of the other. This solution set is plotted in Fig. 3(a), along with its analogous graph, 3(b), reported in Ref.[12]. Figures 2(b) and 3(b) can be obtained by rearranging one of the coupled Lorentzian equations such that it is equal to one of the variables $P_{1,2}$, and substituting this into the second of the Lorentzian equations.

It is possible, for all graphs of Figs. 2 and 3, to isolate the symmetry-broken solution curves using the full equation describing the solution set divided by the equation describing the symmetric solution set followed by simplifications. By studying each component individually, the mathematical analysis can, in some cases, be simplified.

Many of the applications described previously require careful predictions about the characteristics of the symmetry broken region. Some of these characteristics, such as the minimum detuning required for symmetric bistability, or the possibility for symmetry-broken solutions, have been reported for specific values of $A$ and $B: A=1, B=2$ in the case of Ref. [8]. A larger, but finite, range is analysed in Ref. [23, 24], but there appears to be no general analysis spanning all values of $A, B \in \mathbb{R}$. We present this general analysis here along with useful results that are pertinent to the applications mentioned above. Firstly, however, a more immediate question presents itself: which values of $A$ and $B$ are physically feasible?

In the case of two coupled Lorentzian equations describing two counter-propagating fields, the symmetry breaking is a result of the formation of an index grating in the medium due to the standing wave interference pattern that forms [13-16]. In this case, the values that $A$ and $B$ can take are given by $A=1, B=1+h$, where $0 \leq h \leq 1$, depending on the medium's ability to 'wash out' the grating via, for example, diffusion, in the case of a gas or liquid. In a medium with

\begin{tabular}{lcc}
\hline Two counter-propagating fields & A & B \\
\hline Solids (without diffusion) & 1 & 2 \\
General diffusive effects & 1 & $\begin{array}{c}1+h \\
(0<h \leq 1)\end{array}$ \\
Gases (high rates of diffusion) & 1 & $\rightarrow 1$ \\
\hline Two copropagating polarisations & \multicolumn{2}{c}{$A+B=2$} \\
\hline Isotropic media & $2 / 3$ & $4 / 3$ \\
Non-resonant electronic response & $1 / 4$ & $7 / 4$ \\
Liquids, or molecular orientation & 1 & 1 \\
Electrostriction & Wide range of values of $B / A$ \\
$\chi^{(2)}$ media with effective $\chi^{(3)}$ & Wide range of values of $B / A$ \\
Atomic vapours & & \\
\hline Bose-Einstein condensates & Wide range of values of $B / A$ \\
\hline Spin-orbit-coupled BEC
\end{tabular}

TABLE I: A selection of different experimentally-accessible values of $A$ and $B$ for the two set-ups described in Section I.

no diffusive effects, $h=1$, whilst for a highly mobile Kerr medium, such as a gas, $h \rightarrow 0$.

The polarisation case has far greater variation in the possible values that the coupling constants can take. In this case, $A$ and $B$ are related to the third-order nonlinear susceptibility tensor, $\chi^{(3)}$, by

$$
A=\frac{\chi_{1122}^{(3)}+\chi_{1212}^{(3)}}{\chi_{1111}^{(3)}}, \quad B=\frac{\chi_{1122}^{(3)}+\chi_{1212}^{(3)}+2 \chi_{1221}^{(3)}}{\chi_{1111}^{(3)}},
$$

with the constraint that, for CW pumps, $A+B=2$ for an isotropic medium with neglected dispersion [6]. The other cases are: a nonresonant electronic response, $A=2 / 3, B=$ 4/3; liquids or molecular orientation, $A=1 / 4, B=7 / 4$; and electrostriction, $A=1, B=1$ [25]. Deviating momentarily from Kerr media, atomic vapours are likely to show phenomena offering a wide range of possible magnitudes of $A$ and $B[6,26]$, experimentally shown in Ref. [27]. These atomic vapours could be used, for example, in hollow fibres [28]. Modification of an effective $\chi^{(3)}$ nonlinearity has been demonstrated in periodically-poled lithium niobate (PPLN) $[29,30]$ and bismuth borate [31]; cascaded quadratic nonlinearities have also been used to realise a negative effective nonlinear coefficient in the context of self-phase modulation [32-34]. We speculate that it may be possible to generate $B / A<0$ through appropriate material engineering - as considered, for example, in Ref. [35]. Self- and cross-phase modulation coefficients with opposite sign are used in spin-orbit-coupled Bose-Einstein condensates (BEC) [36]. These $A$ and $B$ values are summarised in Table. I.

The values of these coupling constants may not be purely limited to those suggested here. For example, nonlinear thermal effects [37] act to rescale $A$ and $B$ by equal amounts - i.e., they are symmetric effects. The following analysis can be applied to any system described by coupled LLEs or Lorentzian equations of the forms given by Eq. (1) and Eq. (2), respectively, such as in Ref. [24], where both electric and magnetic nonlinearities are modelled. Further systems that exhibit similar symmetry breakings can be found in Refs. [38-41]. 


\section{CHANGING THE RELATIVE STRENGTHS OF SELF- AND CROSS-PHASE MODULATION}

The first generalised result observed here is the region of optical bistability for symmetric solutions, previously seen highlighted in Fig. 1(b) with a red ring. The symmetric solution line in the circulating powers vs. input power diagrams is given by

$$
I=P_{1,2}\left\{1+\left[\theta-(A+B) P_{1,2}\right]^{2}\right\},
$$

The bistable region is found to be bounded by the following:

$$
P_{1,2}=\frac{2 \theta \pm \sqrt{\theta^{2}-3}}{3(A+B)}
$$

where $d I / d\left(P_{1,2}\right)=0$. This reveals that there is a limiting detuning value for symmetric optical bistability of $\theta=\sqrt{3}$ that is independent of the values of the coupling constants. The coupled powers themselves, however, are dependent on the coupling constants. Inserting Eq. (7) into Eq. (6) gives the limits on the input power, between which lies the region of symmetric bistability,

$$
I=-\frac{2\left(2 \theta \pm \sqrt{\theta^{2}-3}\right)\left(-\theta^{2} \pm \theta \sqrt{\theta^{2}-3}-3\right)}{27(A+B)} .
$$

These pump power limits are also dependent on $A$ and $B$, with higher values of $A+B$ leading to a lower value of required input power. Note that, in Eq. (8), a choice of one \pm sign enforces the same choice on the other. A graphical example of these results is given in Fig. 4.

The characteristics of the symmetry-broken region are most easily analysed by examining the symmetry-broken part of Eq. (3), which is given by

$$
\left[\theta-A\left(P_{1}+P_{2}\right)\right]^{2}-P_{1} P_{2}(B-A)^{2}=-1 .
$$

This equation reveals several important conditions which are required for asymmetric solutions to occur. For example, there exists a minimum cavity detuning limit, below which the symmetry broken region will never emerge, for any pump power.

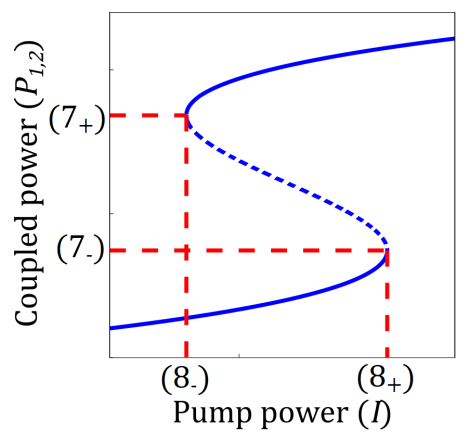

FIG. 4: Example optical bistability of Eq. (6), with limits calculated via Eqs. (7) and (8). Blue dashed line indicates an unstable state, see section IV.
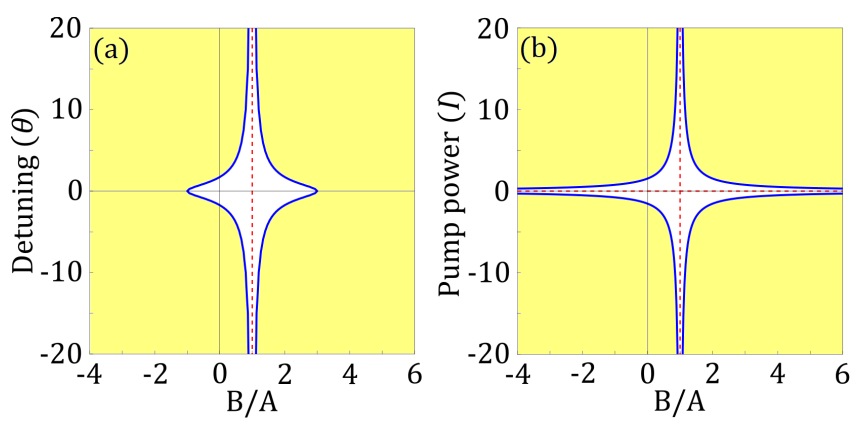

FIG. 5: (a) Minimum detuning required to observe symmetry breaking when changing the ratio of the coupling constants $B / A$. The yellow regions indicate where symmetry breaking is possible, with the blue lines indicating the limits where symmetry breaking becomes impossible. (b) Minimum input power required to observe symmetry breaking. The negative values for $I$ are included for mathematical symmetry only - they are clearly not physically attainable.

This detuning limit is given by

$$
|\theta|>\frac{\sqrt{\left(3-\frac{B}{A}\right)\left(1+\frac{B}{A}\right)}}{\left|\frac{B}{A}-1\right|} .
$$

As shown in Fig. 5a, this limit equation yields itself some interesting results. The first one is that, for a unity ratio between the two coupling constants, symmetry breaking is never possible, since $\theta_{\min }$ diverges to $\infty$. The second interesting result is that, for $B / A>3$ or $B / A<-1$, symmetry breaking is attainable for all detuning values, even $\theta=0$, for pump powers above given thresholds.

Similar to Eq. (10), an equation defining the pump power limit is also derivable and is given by

$$
|I|>\frac{\frac{8}{9} \sqrt{3}}{\left|\frac{B}{A}-1\right|} .
$$

Below this limit, symmetry breaking is again not possible for any range of cavity detunings. Unlike with the detuning limit, this power limit only falls to 0 as $B / A$ tends to $\infty$.

Further analysis of Eq. (9) reveals the coupled powers at which the symmetry breaking bifurcation points are located. These points, where the symmetry-broken region opens/closes, are given by

$$
P_{1,2}=C \pm \frac{D}{(3 A-B)(A+B)},
$$

where

$$
C=\frac{2 A \theta}{(3 A-B)(A+B)},
$$

and

$$
D=\sqrt{-3 A^{2}+\theta^{2}(A-B)^{2}-2 A B+B^{2}} .
$$

A final result of interest emerging from Eq. (9) is of importance for the optimisation of the formation of isolators for integrated photonic circuits, such as in Ref. [10]. For such applications, one mode must be suppressed as much as possible, 
whilst the other mode is maximised. The coupled powers of the greatest possible difference are given by:

$$
P_{1,2}=C \pm \frac{1}{B-A} \frac{D}{\sqrt{(3 A-B)(A+B)}} .
$$

These special points are all summarised in Fig. 2(a) (points a, $\mathrm{b}, \mathrm{c}, \mathrm{d}, \mathrm{e})$ with the input power required to reach each point given by substituting the appropriate equations into Eq. (2).

Eq. (12) identifies critical values for the 'bursting' of the bubble, beyond which the symmetry-broken region opens, but never closes. Consequently, isolators based on this principle would have no upper limit of operational power (above which they would return to symmetric solutions). These bursting ratios, beyond which the symmetry-broken solution forms a parabola rather than an ellipse, are given by $B / A>3$ and $B / A<-1$.

Turning attention to Eq. (4), some key points of the detuning scans can be identified. At the power limit, Eq. (11), the symmetry-broken region emerges at

$$
P_{1,2}=\frac{3}{4} I
$$

while the symmetry breaking bifurcation point pair is given by solving the real roots to the quartic equation

$$
P_{1,2}^{4}-I P_{1,2}^{3}+\left[\frac{I}{2(A-B)}\right]^{2}=0 .
$$

The detuning requirements to observe these points can then be obtained by substituting the appropriate equations into Eq. (2). These special points are shown in Fig. 3(a) (points $\mathrm{f}, \mathrm{g}, \mathrm{h}$ ).

In closing this section, we note that the value of $B / A$ also affects where the symmetry-broken solution line appears with respect to the bistable symmetric solution line. It is known that, for $A=1$ and $B=2$, the symmetry-broken 'bubble' appears on the upper branch of the bistable symmetric solution line for graphs like that of Fig. 1(b) [8]. This is because, for this $B / A$ ratio, Eq. (10) dictates that symmetry-broken solutions are only possible for $\theta \geq \sqrt{3}$, with $\theta=\sqrt{3}$ being the condition where optical bistability emerges. This holds true for any $1<B / A \leq 2$. Above ratios of 2 , the minimum detuning for symmetry breaking is below that for optical bistability, meaning that it is now possible to observe the symmetrybroken solutions without bistability, Fig. 6(a). More interesting is the region $B / A<1$. For $0<B / A<1$, symmetry breaking is again only possible for detunings above the $\sqrt{3}$ value for optical bistability, but now the symmetry-broken bubble appears on the middle branch of the bistable region, as shown in Fig. 6(b). Progressing further, for $B / A<0$, it is once again possible to observe the symmetry-broken solutions for detunings lower than the minimum required for symmetric solution line optical bistability.

We note that when plotting in the style of Fig. 1(a) for $B=0$, it would appear that symmetry broken solutions are, interestingly, still possible, as shown in Fig. 6(c). This explains the continuous nature of all equations described previously, and Fig. 5, about $B / A=0$. In this case the appearance (a)

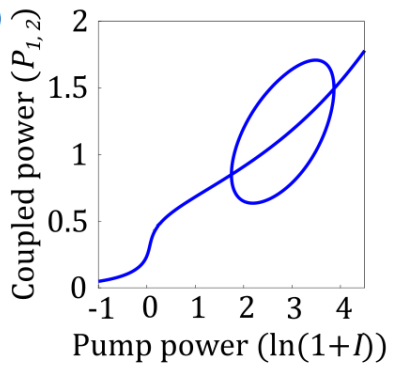

(b)

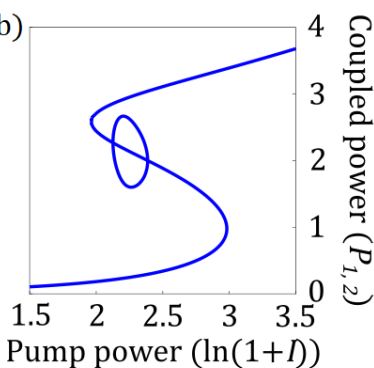

(d)

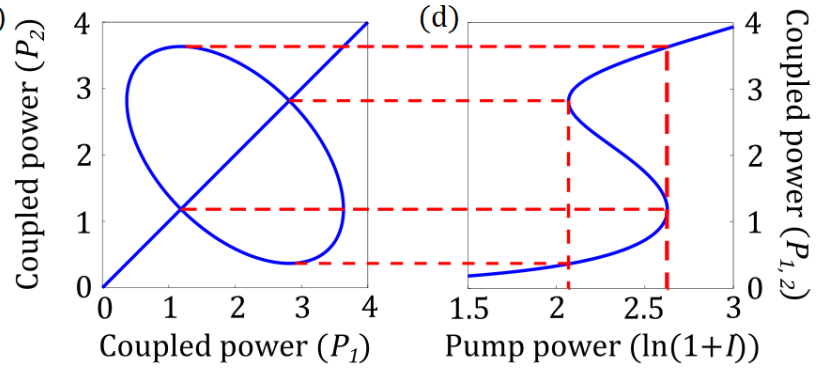

FIG. 6: (a), (b) Plots of the input power scans for $B / A=2.2, \theta=1.5$ and $B / A=0.5, \theta=4$ respectively. (c), (d) show the special case of $B=0, A \in \mathbb{R}$, with $A=1, \theta=3$. (c) shows the possibility still for symmetry-broken solutions, while (d) shows how their origin is due to the bistable region of the Lorentzian equation.

of the apparently symmetry-broken solutions is due to the imposed constraint that both $\theta$ and $I$ are equal for both equations. This results in the two, now uncoupled, Lorentzian equations being identical. The 'symmetry-broken' solutions arise physically from the possibility of one field being on the top branch of the optical bistability while, simultaneously, the other is on the bottom, or vice versa, see Fig. 6(d).

\section{GENERALISED STABILITY ANALYSIS}

In the same spirit as in Ref. [12], we recognise that Eq. (2) is the steady state of the following time-dependent system:

$$
\frac{\partial E_{ \pm}}{\partial t}=E_{\text {in }}-\left[1+i\left(\theta-A\left|E_{ \pm}\right|^{2}-B\left|E_{\mp}\right|^{2}\right)\right] E_{ \pm} .
$$

Following the procedure set out in Ref. [12], we add small perturbations to the steady state solution, calculate the eigenvalues of the (Jacobian) matrix that results, and assess the stability of this system. The eigenvalues of the linear stability of Eq. (18) have the same form as those provided in Ref. [12]:

$$
\lambda=-1 \pm \sqrt{\frac{-\alpha_{1} \beta_{1}-\alpha_{2} \beta_{2} \pm S}{2}},
$$

with

$$
S=\sqrt{\left(\alpha_{1} \beta_{1}-\alpha_{2} \beta_{2}\right)^{2}+4 \alpha_{1} \alpha_{2} \gamma^{2}}
$$

but the quantities $\alpha_{1,2}, \beta_{1,2}$ and $\gamma^{2}$ take on forms generalised to arbitrary self- and cross-phase modulation coefficients: $\alpha_{1,2}=$ $\theta-A P_{1,2}-B P_{2,1}, \beta_{1,2}=\theta-3 A P_{1,2}-B P_{2,1}$, and $\gamma^{2}=4 B^{2} P_{1} P_{2}$. 
Note that, in Eq. (19), one \pm choice enforces no restrictions on the other \pm , giving a total of four eigenvalues. The the quantity $S$ plays an essential role in establishing the stability of the system. If $S$ is real, and the quantity under the square root in Eq. (19) is negative for both $\pm S$, i.e, $S<\alpha_{1} \beta_{1}+\alpha_{2} \beta_{2}$, then all the eigenvalues are complex numbers with real part equal to -1 , leading to full stability of the corresponding stationary states. On the other hand, if $S$ is real, and the quantity under the square root in Eq. (19) is positive, then one real eigenvalue can be positive (meaning a non-oscillatory instability) if

$$
S>2+\alpha_{1} \beta_{1}+\alpha_{2} \beta_{2},
$$

with the maximum of two real eigenvalues being positive when

$$
S<-\left(2+\alpha_{1} \beta_{1}+\alpha_{2} \beta_{2}\right)
$$

is also satisfied. Note that this condition for a second unstable eigenvalue is only possible when $2+\alpha_{1} \beta_{1}+\alpha_{2} \beta_{2}<0$.

Under the condition of $S$ being purely imaginary, the eigenvalues, Eq. (19), are complex with the real $(R)$ and imaginary $(\Omega)$ parts, corresponding to the growth rate and the angular frequency respectively. These have the structure of those given in Ref. [12].

The instabilities are then obtained by finding the conditions for which $R>0$, and correspond to

$$
\left|S^{2}\right|>8\left(2+\alpha_{1} \beta_{1}+\alpha_{2} \beta_{2}\right) .
$$

This birth of a stable limit cycle implies a supercritical Hopf bifurcation. Note that, for this system, if we have a pair of oscillatory eigenvalues with positive real part, then the real part of the remaining two must necessarily be negative. Interestingly, oscillatory instabilities can only appear in the symmetry-broken branches of the stationary solutions, regardless of $B / A$; no oscillatory instability can be found on the symmetric branches, since, in this case, $S$ is always a real number.

By evaluating partial derivatives with respect to the detunings and pump powers, we can also locate the generalised condition for a transcritical bifurcation that occurs during a scan of the common-mode detuning under imbalanced conditions. This point is given by:

$$
\frac{1+\alpha_{1}^{2}}{2 P_{1} \alpha_{1}}=\frac{1+\alpha_{2}^{2}}{2 P_{2} \alpha_{2}}=A-B .
$$

This condition is the generalized form of that presented in Ref. [12] for $A=1$ and $B=2$.

Real eigenvalue instabilities can be found on the symmetric branches of the stationary solutions, where $\alpha=\alpha_{1}=\alpha_{2}$ and $\beta=\beta_{1}=\beta_{2}$. Here, real $S$ means $S=2|\gamma \alpha|$ and the conditions (21)-(22) reduce to

$$
|\gamma \alpha|>1+\alpha \beta, \text { and }|\gamma \alpha|<-(1+\alpha \beta) .
$$

On the symmetric branches, the bifurcations corresponding to conditions (25) are either the saddle-node bifurcations of the S-shaped stationary curves or the pitchfork bifurcations leading to symmetry-breaking solutions.
To illustrate the effect of the cross-phase modulation coefficient on the stability of the system, we report here about two limit cases of small and large cross-phase to self-phase modulation ratio, $B / A$. Complex eigenvalues with positive real part may lead to experimentally-accessible oscillations, since their amplitude will eventually stop growing due to saturation effects that are not captured by the above stability analysis.

Figure 7 illustrates stable, unstable, and oscillatory unstable regimes for a variety of choices of parameters for a small value of $B / A=0.9$, where the self-phase modulation is stronger than the cross-phase modulation. In this regime, the system is not strongly susceptible to either symmetry breaking or the onset of growing oscillations, and so the power thresholds for accessing these phenomena are very high. When increasing the input power, I, symmetry-broken solutions occur in the middle branch of the bistable S-shaped curves. Some of these solutions later gain stability, and others exhibit growing oscillations; the system begins to display multi-stability of symmetric and asymmetric solutions, as observed in Figs. 7 (c), (d), (g), and (h).

For larger values of $B / A$ such as $B / A=7$, large parameter regions where stationary states are susceptible to oscillations are observed, as displayed in the detuning scan in Fig. 8 for $I=3.3$. Figure 8 is also consistent with a prediction made in Section III: symmetry-broken solutions at zero detuning.

\section{TEMPORAL DYNAMICS}

The stability analysis of the previous section described how the system responds to small, noise-like, perturbations with respect to changes of the ratio $B / A$. In this section, we investigate the full temporal evolutions and oscillations by using numerical integration of Eq. (18). The temporal dynamics of optical systems can lead to a range of applications, such as polarization scramblers or devices with periodic switching of polarisation state or direction. The dynamics of similar systems to those described here being previously studied in Ref. [28]. These numerical integrations illustrate the consequences of modifying the relative strengths of self- and cross-phase modulation for the onset and extent of deterministic chaos. We consider changes in the cross- to self-phase modulation ratio $B / A$ and found that increasing $B / A$ increases the susceptibility of the system to temporal instabilities and chaos. For each parameter configuration specified by $B, \theta$, and $I$ in the oscillatory regime, we sample the evolution trajectories of the coupled powers $P_{1,2}$ by evaluating the Poincare section corresponding to their local maxima where the first derivative in time is zero and second derivative is negative. In this way, we can monitor the number of maxima per period and register their values. Fig. 9(a) shows the maxima of the coupled power $P_{1}$ during oscillations when changing $B$ from 1.5 to 7 , for $A=1, \theta=5$, and $I=3.3$. We observe sequences of bifurcations, chaotic windows and sudden crises. The power ranges spanned by the oscillations clearly increase with the cross-phase modulation magnitude.

To illustrate the susceptibility of the system to temporal oscillations at large values of $B / A$, we show in Fig. 9(b) 

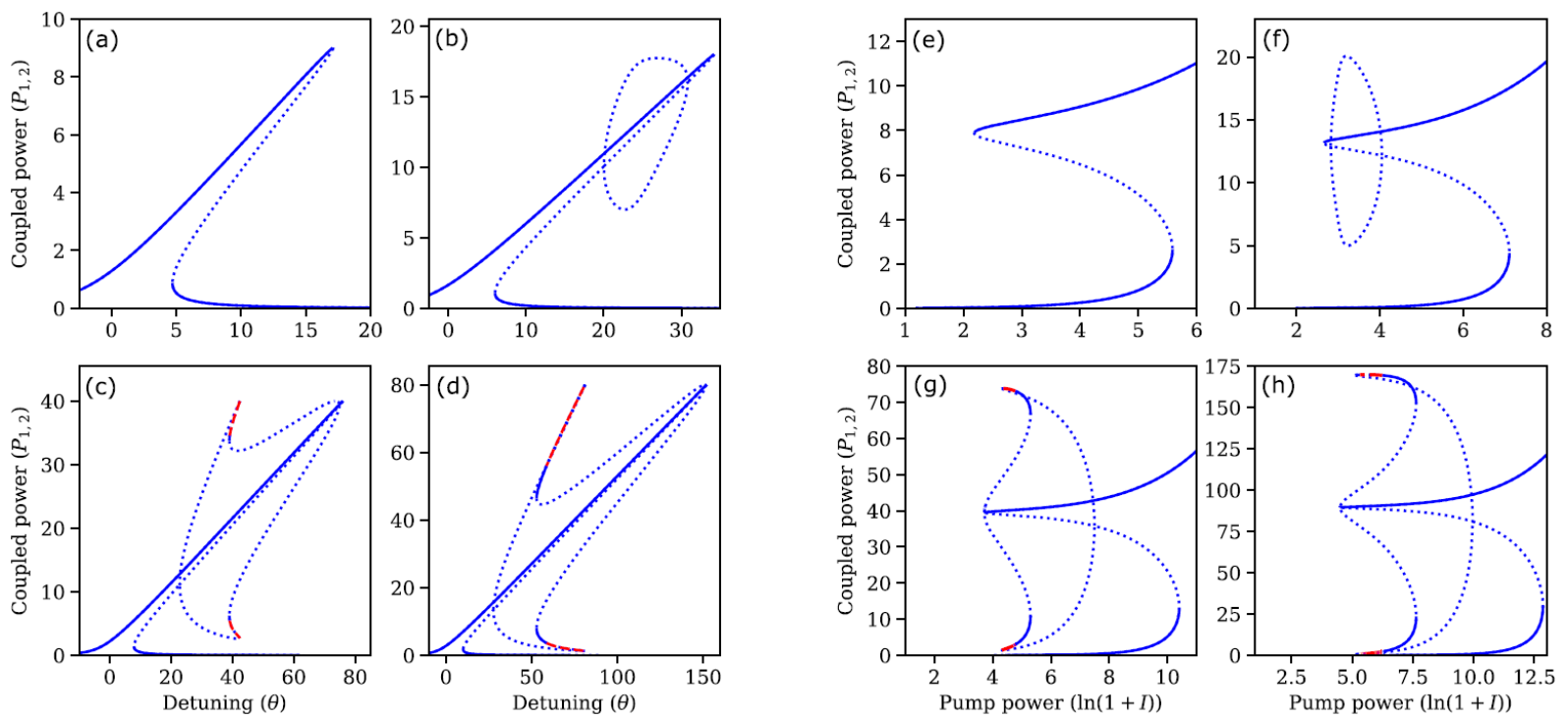

FIG. 7: (a)-(d) illustrate the coupled power $P_{1,2}$, against the detuning parameter, $\theta$ for $A=1$ and $B=0.9$. Stable and non-oscillatory unstable solutions are shown in solid and dotted blue, respectively, whilst oscillatory instabilities are shown in dashed red. The input power, $I$, increases with each frame. (a) $I=9$. No symmetry-broken solutions have yet emerged. (b) $I=18$. Spontaneous symmetry breaking occurs in the unstable symmetric branch. (c) $I=40$. Stable symmetry-broken solutions emerge but quickly lose stability to complex eigenvalues with positive real parts. (d) $I=80$. The entire structure is stretched, including the region of unstable oscillatory and stable symmetry-broken solutions. Note the parameter range with four stable solutions. The right-hand plots illustrate the coupled power, $P_{1,2}$, against the input power, $I$. The key phenomena shown on the left are visible here. (e) $\theta=15$. (f) $\theta=25$. (g) $\theta=75$. (h) $\theta=170$. Note that, in the above plots, growing oscillations are always accompanied by a stable solution so these oscillations may not be experimentally observable.

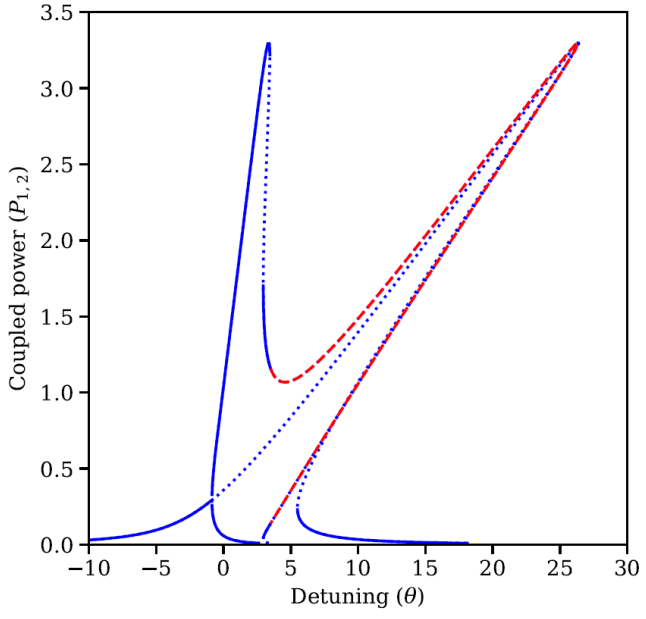

FIG. 8: Coupled power $P_{1,2}$, against the detuning parameter, $\theta$ for $A=1, B=7$ and $I=3.3$. Stable and non-oscillatory unstable solutions are shown in solid and dotted blue, respectively, whilst oscillatory instabilities are shown in dashed red.

the Poincaré sections in a detuning scan for $B / A=7$ and $I=3.3$. These are the same parameters of the stationary solution curves displayed in Fig. 8. In this case, the symmetry breaking bifurcation occurs at negative values of the detuning $\theta$. After this bifurcation, one of the coupled powers increases while the other decreases. The onset of oscillations occurs when the decreasing coupled power approaches zero (just after $\theta=3.5$ ) in a region without stable stationary states. Windows of periodic and chaotic oscillations alternate with increasing detunings until no symmetry-broken solutions are observed just after $\theta=5.74$. For large detunings and close to the end of the dynamical regimes, we observe a region of coexistence of a stable symmetric stationary state (black solid line in Fig. 9(b)) and chaotic oscillations. Similar coexistence regions are also found for lower values of the parameter $B / A$.

The richness of oscillatory behaviour for $B / A=7$ and $I=3.3$ is presented in Fig. 10, which shows specific cases of different oscillatory regimes for given values of the detuning, as predicted by Fig. 9(b). Figure 10(a),(b) show periodic oscillations close to the onset of temporal instability. Each asymmetrically coupled power has undergone a Hopf bifurcation, leading to a small amplitude modulation. The dynamical behaviour is attracted to two disjointed regions of the phase space. When increasing the detuning, the amplitude of the oscillations grows and chaotic dynamics are observed (see Fig. 10(c),(d)). We note, however, that the oscillations now switch erratically from one dominant field to the other and that the attractor covers a single region of the phase space for both coupled fields. This latter aspect becomes even more striking by a further increase in the detuning parameter as shown in Fig. 10(e),(f). Here, the system displays a periodic switching between the two modes and the projection of the attractors of the two fields overlap completely. An effect such as this has potential application in photonic systems where control of the output pulses, in particular of their polarization or propagation direction, is required. While we show this 

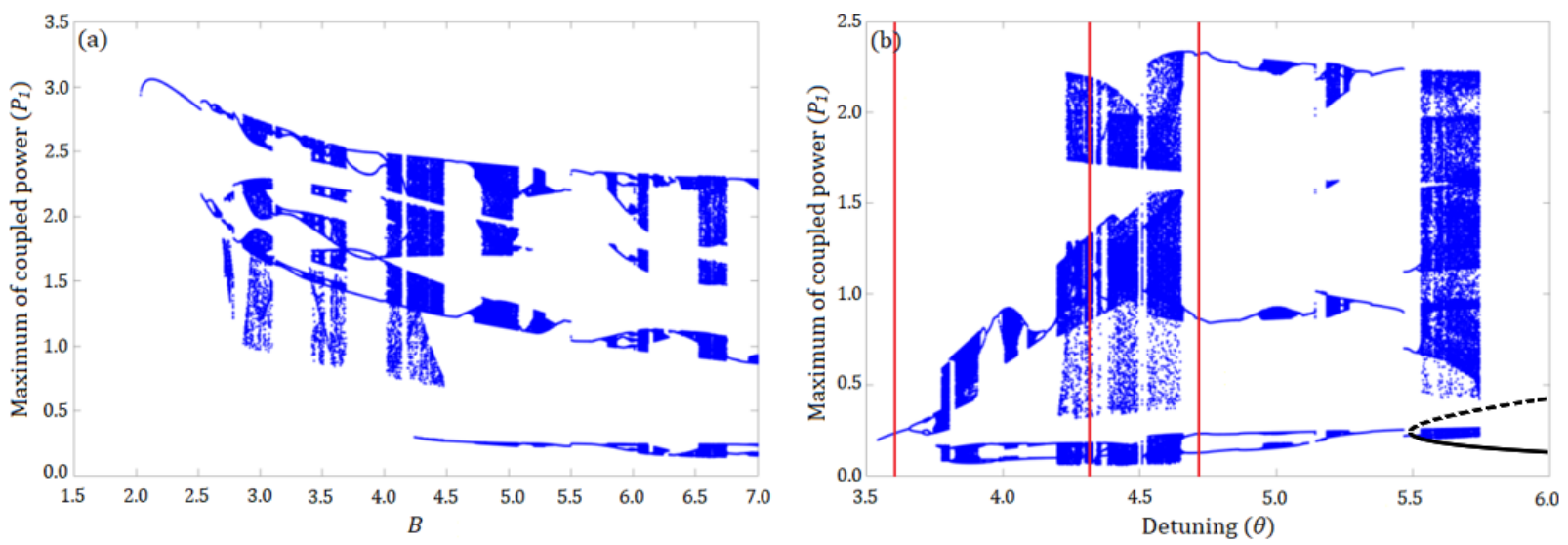

FIG. 9: (a) Poincare sections of the maxima of oscillating coupled power $P_{1}$, versus the cross-phase modulation coefficient, $B$, for constant $A=1$. These points corresponds to constant values of the detuning $\theta=5$ and input power $I=3.3$. (b) Illustration of periodic oscillations and deterministic chaos in the Poincaré sections of the maxima of $P_{1}$ when varying the detuning parameter, $\theta$ for a large cross- to self-phase modulation ratio. In this case, $A=1, B=7$ and the input power is $I=3.3$. Note the dense columns of chaotic windows. The solid (dashed) black line corresponds to a stable (unstable) symmetric stationary state. The red lines indicate the detuning values used in Fig. 10.
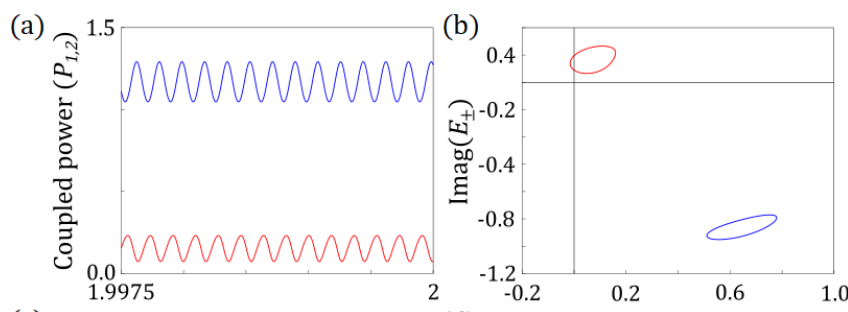

(c)
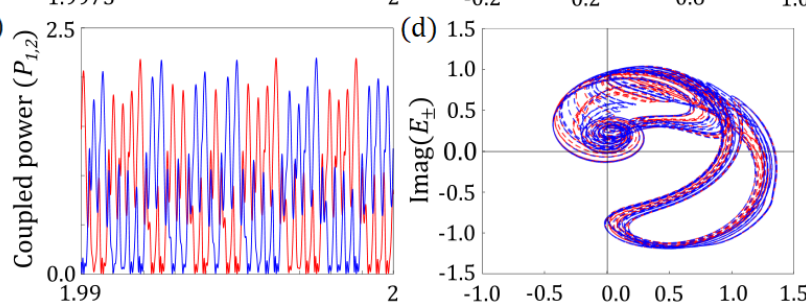

(e)
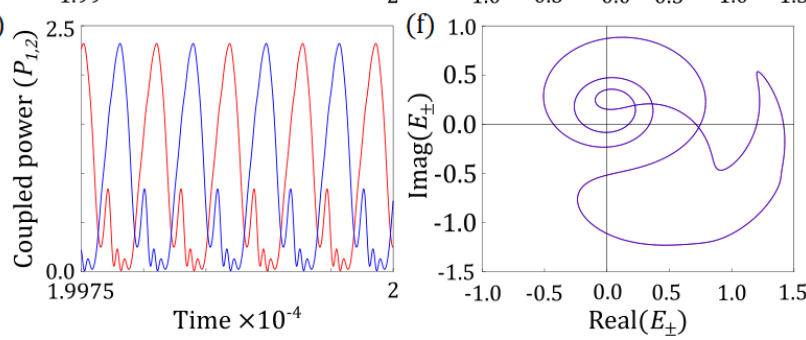

FIG. 10: Results of numerical simulations of Eq. (18) for $A=1$, $B=7$, and $I=3.3$ showing oscillatory (a), (b), chaotic switching (c), (d), and periodic switching (e), (f) solutions. (a), (c) and (e) all show the temporal evolutions of the coupled powers while (b), (d) and (e) show the phase space orbits of the real and imaginary components of the late temporal evolutions of $E_{ \pm}$. The detuning parameter for (a), (b) is $\theta=3.6$, for (c), (d) is $\theta=4.31$ and for (e), (f) is $\theta=4.755$. Time is dimensionless.

behaviour for $B / A=7$, we also predict that it would be present for many other values of the self- and cross-phase modulation constants.

\section{CONCLUSION}

We have presented a theoretical model for the spontaneous symmetry breaking of light in ring resonators, generalised to arbitrary strengths of self- and cross-phase modulation, and describing the coupling of either two circularly-polarised or two counter-propagating fields. We have presented the characteristics of the steady-state symmetry-broken region, such as the minimum criteria for its observation, its opening and closing bifurcation points and the conditions for maximum difference in the coupled intensities. It was observed how the position of the symmetry-broken region varies with respect to the symmetric optical bistability, along with the dependence of the oscillatory regime on the value of $B / A$. Finally, we have shown the presence of a subset of oscillatory solutions which may lead to new applications such as sequences of pulses with given polarization or propagation direction. These oscillatory behaviours include different styles of switching between modes. Periodic switching suggests a partial symmetry restoration in a chaotic regime. These findings should be applicable to a large range of experimental settings featuring nonlinear media, including Kerr liquids and atomic vapours, as well as situations that exhibit variable overlap (and, hence, variable cross-phase modulation) between two optical modes.

\section{ACKNOWLEDGEMENTS}

The authors would very much like to thank Jonathan M. Silver and Leonardo Del Bino for useful and stimulating discussions. We also acknowledge financial support from: EPSRC DTA Grant No. EP/M506643/1; H2020 Marie SklodowskaCurie Actions (MSCA) (748519, CoLiDR); National Physical Laboratory Strategic Research; H2020 European Research Council (ERC) (756966, CounterLight); Engineering and Physical Sciences Research Council (EPSRC). 
[1] L. A. Lugiato and R. Lefever, "Spatial dissipative structures in passive optical systems," Physical Review Letters, vol. 58, p. 2209, 1987.

[2] Y. K. Chembo, D. Gomila, M. Tlidi, and C. R. Menyuk, "Topical issue: Theory and applications of the Lugiato-Lefever equation," European Physical Journal D, vol. 71, p. 299 and following articles, 2017.

[3] M. Haelterman, S. Trillo, and S. Wabnitz, "Dissipative modulation instability in a nonlinear dispersive ring cavity," Optics Communications, vol. 91, pp. 401-407, 1992.

[4] L. Lugiato, F. Prati, and M. Brambilla, Nonlinear optical systems. Cambridge University Press, 2015.

[5] F. Castelli, M. Brambilla, A. Gatti, F. Prati, and L. A. Lugiato, "The LLE, pattern formation and a novel coherent source," The European Physical Journal D, vol. 71, p. 84, 2017.

[6] J. Geddes, J. Moloney, E. Wright, and W. Firth, "Polarisation patterns in a nonlinear cavity," Optics Communications, vol. 111, pp. 623-631, 1994.

[7] A. Kaplan and P. Meystre, "Enhancement of the Sagnac effect due to nonlinearly induced nonreciprocity," Optics Letters, vol. 6, pp. 590-592, 1981 .

[8] A. Kaplan and P. Meystre, "Directionally asymmetrical bistability in a symmetrically pumped nonlinear ring interferometer," Optics Communications, vol. 40, pp. 229-232, 1982.

[9] L. Del Bino, J. M. Silver, S. L. Stebbings, and P. Del'Haye, "Symmetry breaking of counter-propagating light in a nonlinear resonator," Scientific Reports, vol. 7, p. 43142, 2017.

[10] L. Del Bino, J. M. Silver, M. T. M. Woodley, S. L. Stebbings, X. Zhao, and P. Del'Haye, "Microresonator isolators and circulators based on the intrinsic nonreciprocity of the Kerr effect," Optica, vol. 5, pp. 279-282, 2018.

[11] E. Wright, P. Meystre, W. Firth, and A. Kaplan, "Theory of the nonlinear Sagnac effect in a fiber-optic gyroscope," Physical Review A, vol. 32, p. 2857, 1985.

[12] M. T. M. Woodley, J. M. Silver, L. Hill, F. Copie, L. Del Bino, S. Zhang, G.-L. Oppo, and P. Del'Haye, "Universal symmetrybreaking dynamics for the Kerr interaction of counterpropagating light in dielectric ring resonators," Physical Review A, vol. 98, p. 053863, 2018.

[13] K. Otsuka, "Nonlinear antiresonant ring interferometer," Optics Letters, vol. 8, pp. 471-473, 1983.

[14] W. Firth, I. Galbraith, and E. Wright, "Diffusion and diffraction in dispersive optical bistability," Journal of the Optical Society of America B, vol. 2, pp. 1005-1009, 1985.

[15] W. Firth and C. Paré, "Transverse modulational instabilities for counterpropagating beams in Kerr media," Optics Letters, vol. 13, pp. 1096-1098, 1988.

[16] W. Firth, A. Fitzgerald, and C. Paré, "Transverse instabilities due to counterpropagation in Kerr media," Journal of the Optical Society of America B, vol. 7, pp. 1087-1097, 1990.

[17] C. Wang and C. Search, "A Nonlinear Microresonator Refractive Index Sensor," Journal of Lightwave Technology, vol. 33, pp. 4360-4366, 2015.

[18] S. Coen and M. Erkintalo, "Temporal cavity solitons in Kerr media", in Nonlinear Optical Cavity Dynamics: From Microresonators to Fiber Lasers. edited by P. Grelu (Wiley, Weinheim, Germany), pp 11-40, 2016.

[19] R. Gallego, M. San Miguel, and R. Toral, "Self-similar domain growth, localized structures, and labyrinthine patterns in vectorial Kerr resonators," Physical Review E, vol. 61, pp. 22412244, 2000.
[20] F. Copie, M. T. Woodley, L. D. Bino, J. M. Silver, S. Zhang, and P. Del'Haye, "Interplay of Polarization and Time-Reversal Symmetry Breaking in Synchronously Pumped Ring Resonators," Physical Review Letters, vol. 122, p. 013905, 2019.

[21] B. Garbin, J. Fatome, G.-L. Oppo, S. G. Murdoch, M. Erkintalo, and S. Coen, "Symmetry breaking: balancing asymmetries," in 2019 Conference on Lasers and Electro-Optics Europe and European Quantum Electronics Conference, pp. EF2-3, Optical Society of America, 2019.

[22] B. Garbin, J. Fatome, G.-L. Oppo, M. Erkintalo, S. G. Murdoch, and S. Coen, "Asymmetric balance in symmetry breaking," arXiv:1904.07222, 2019.

[23] D. A. Mártin and M. Hoyuelos, "Codimension 2 and 3 situations in a ring cavity with elliptically polarized electromagnetic waves," Physica D, vol. 259, pp. 37-47, 2013.

[24] D. A. Mártin and M. Hoyuelos, "Homogeneous solutions for elliptically polarized light in a cavity containing materials with electric and magnetic nonlinearities," Physical Review A, vol. 82, p. 033841, 2010.

[25] R. W. Boyd, Nonlinear optics. Elsevier, 2003.

[26] J. B. Geddes, "Patterns, fishing and nonlinear optics.," PhD thesis, The University of Arizona, 1994.

[27] J. Burgin, C. Guillon, and P. Langot, "Femtosecond investigation of the non-instantaneous third-order nonlinear suceptibility in liquids and glasses," Applied Physics Letters, vol. 87, p. 211916,2005

[28] M. Schmidberger, D. Novoa, F. Biancalana, P. S. J. Russell, and N. Joly, "Multistability and spontaneous breaking in pulseshape symmetry in fiber ring cavities," Optics Express, vol. 22, pp. 3045-3053, 2014.

[29] S. Das, S. Mukhopadhyay, N. Sinha, A. Saha, P. Datta, and S. Saltiel, "Modulation of effective nonlinearity $(\chi(3))$ due to cascaded processes in PPLN for direct third harmonic generation," in Lasers and Electro-Optics, 2006 and 2006 Quantum Electronics and Laser Science Conference. CLEO/QELS 2006. Conference on, pp. 1-2, IEEE, 2006.

[30] O. Balachninaite, R. Grigonis, A. Piskarskas, V. Sirutkaitis, and R. Eckardt, "Self-and cross-phase modulation in a PPLN OPO," in Conference on Lasers and Electro-Optics, p. CThC7, Optical Society of America, 2000.

[31] K. Miyata, N. Umemura, and K. Kato, "Phase-matched pure $\chi$ (3) third-harmonic generation in noncentrosymmetric BiB3O6," Optics Letters, vol. 34, pp. 500-502, 2009.

[32] A. S. Mayer, C. R. Phillips, and U. Keller, "Watt-level 10gigahertz solid-state laser enabled by self-defocusing nonlinearities in an aperiodically poled crystal," Nature Communications, vol. 8, p. 1673, 2017.

[33] C.-W. Chen, J.-L. Tang, K.-H. Chung, T.-H. Wei, and T.-H. Huang, "Negative nonlinear refraction obtained with ultrashort laser pulses," Optics Express, vol. 15, p. 7006, 2007.

[34] F. Saltarelli, A. Diebold, I. J. Graumann, C. R. Phillips, and U. Keller, "Self-phase modulation cancellation in a high-power ultrafast thin-disk laser oscillator," Optica, vol. 5, p. 1603, 2018.

[35] Q. Pan, W. Chung, and K. Chow, "The coupled Hirota system as an example displaying discrete breathers: Rogue waves, modulation instability and varying cross-phase modulations," AIP Advances, vol. 8, p. 095303, 2018.

[36] Y. Li, Z. Luo, Y. Liu, Z. Chen, C. Huang, S. Fu, H. Tan, and B. A. Malomed, "Two-dimensional solitons and quantum droplets supported by competing self-and cross-interactions 
in spin-orbit-coupled condensates," New Journal of Physics, vol. 19, p. 113043, 2017.

[37] T. Carmon, L. Yang, and K. J. Vahala, "Dynamical thermal behavior and thermal self-stability of microcavities," Optics Express, vol. 12, p. 4742, 2004.

[38] A. Armaroli, P. Féron, and Y. Dumeige, "Microwave generation on an optical carrier in microresonator chains," Physical Review A, vol. 98, p. 013848, 2018.

[39] A. Sigler and B. A. Malomed, "Solitary pulses in linearly coupled cubic-quintic Ginzburg-Landau equations," Physica D, vol. 212, pp. 305-316, 2005.

[40] A. Sigler, B. A. Malomed, and D. V. Skryabin, "Localized states in a triangular set of linearly coupled complex GinzburgLandau equations," Physical Review E, vol. 74, p. 066604, 2006.

[41] C. Milián, Y. V. Kartashov, D. V. Skryabin, and L. Torner, "Cavity solitons in a microring dimer with gain and loss," Optics Letters, vol. 43, pp. 979-982, 2018. 